\title{
Some Symmetry Properties of Almost $S$-Manifolds
}

\author{
Yavuz Selim Balkan*, Mehmet Zeki Sarikaya \\ Düzce University, Faculty of Arts and Sciences, Department of Mathematics, Konuralp Campus, Düzce/TURKEY, \\ 03805412404, 03805412403, \\ y.selimbalkan@gmail.com; sarkkayamz@gmail.com \\ *Corresponding author
}

Received: 22 February 2017

Accepted: 19 June 2017

DOI: $10.18466 /$ cbayarfbe.339323

\begin{abstract}
Manifold theory is an important topic in differential geometry. Riemannian manifolds are a wide class of differentiable manifolds. Riemannian manifolds consist of two fundamental class, as contact manifolds and complex manifolds. The notion of globally framed metric $f$-manifold is a generalization of these fundamental classes. Almost $S$-manifolds which are globally framed metric $f$-manifold generalize some contact manifolds carrying their dimension to $(2 n+s)$. On the other hand, classification is important for Riemannian manifolds with respect to some intrinsic and extrinsic tools as well as all sciences. Moreover, symmetric manifolds play an important role in differential geometry. There are a lot of symmetry type for Riemannian manifolds with respect to different arguments. Under these considerations, in the present paper we study some symmetry conditions on almost $S$-manifolds. We investigate weak symmetries and $\varphi$ symmetries of these type manifolds. We obtain some necessary and sufficient conditions to characterize of their structures. Firstly, we prove that the existence of weakly symmetric and weakly Ricci symmetric almost $S$-manifolds under some special conditions. Then, we show that every $\varphi$-symmetric almost $S$-manifold verifying the $(\kappa, \mu)$-nullity distribution is an $\eta$-Einstein manifold of globally framed type. Finally, we get some necessary and sufficient condition for a $\varphi$-Ricci symmetric almost $S$-manifold verifying the $(\kappa, \mu)$ -nullity distribution to be an $\eta$-Einstein manifold of globally framed type.
\end{abstract}

Keywords $-\eta$-Einstein Manifold, $\varphi$-Ricci Symmetric Manifolds, Almost $S$-Manifold, Globally Framed Metric $f$-Manifold, $S$-Structure, Weakly Symmetric Manifold.

\section{Introduction}

An extensive research about contact geometry is done in recent years. In the present paper, we are concerned with weak symmetries and Ricci symmetries of almost $S$-manifolds. We recall the price definitions.

Let $M$ be a $(2 n+s)$-dimensional manifold and $\varphi$ is a non-null $(1,1)$-tensor field on $M$. If $\varphi$ satisfies

$\varphi^{3}+\varphi=0$,

then $\varphi$ is called an $f$-structure and $M$ is called an $f$ -manifold [1].
Let $M$ be a $(2 n+s)$-dimensional manifold. If there are given on $M$ an $f$-structure $\varphi, S$ global vector fields $\xi_{1}, \ldots, \xi_{s}$ and 1 -forms $\eta^{1}, \ldots \eta^{s}$ on $M$ satisfying the $\varphi^{2}=-I d+\sum_{j=1}^{s} \eta^{j} \otimes \xi_{j}$,

following conditions $\varphi \xi_{i}=0, \quad \eta^{j} \circ \varphi=0$,

$$
\eta^{i}\left(\xi_{j}\right)=\delta_{j}{ }^{i} \text {, }
$$

for all $i, j \in\{1, \ldots s\}$, then $\left(\varphi, \xi_{i}, \eta^{i}\right)$ is called a glob- 
ally framed $f$-structure and $M$ is called a globally framed $f$-manifold [2]. On a globally framed $f$-manifold $M$, if there exists a Riemannian metric which satisfies

$$
\begin{aligned}
& g(\varphi X, \varphi Y)=g(X, Y)-\sum_{k=1}^{s} \eta^{k}(X) \eta^{k}(Y), \\
& g\left(X, \xi_{k}\right)=\eta^{k}(X),
\end{aligned}
$$

for all vector fiels $X$ and $Y$ on $M$, then $M$ is called a globally framed metric $f$-manifold [2]. On a globally framed metric $f$-manifold, fundamental 2 -form $\Phi$ is defined by $\Phi(X, Y)=g(X, \varphi Y)$ for all vector fiels $X, Y$ [2]. For a globally framed metric $f$-manifold,

$N_{\varphi}+\sum_{k=1}^{s} d \eta^{k} \otimes \xi_{k}=0$,

is satisfied, then $M$ is called normal globally framed metric $f$-manifold, where $N_{\varphi}$ denotes the Nijenhuis torsion tensor of $\varphi[3]$.

A globally framed metric $f$-manifold is said to be an almost $S$-manifold if and only if $d \eta^{1}=\ldots=d \eta^{s}=\Phi$ and $d \Phi=0$. An almost $S$-manifold which is normal is called $S$-manifold [4].

The study of globally framed metric $f$-manifold was started by Blair [4], Goldberg and Yano [5], Vanzura [6]. Almost $S$-structures were studied, without being precisely named, by Cabrerizo et al. [7]. Then Duggal et al. [8] also studied such manifolds and gave them the name almost $S$ -manifold.

On an almost $S$-manifold, we can define a $(1,1)$ tensor fields

$h_{i}:=\frac{1}{2} L_{\xi_{i}} \varphi$,

for $i=1, \ldots, s$, where $L$ denotes the Lie derivative in [7]. We use extensively the properties of these tensor fields in the present paper. In particular, these operators are self-adjoint, traceless, anticommute with $\varphi$ and, we have

$h_{i} \xi_{j}=0, \quad \eta^{i} \circ h_{j}=0$.

for $i, j \in\{1, \ldots s\}$ [7]. Moreover, the following identities hold
$\nabla_{X} \xi_{i}-\varphi X-\varphi h_{i} X$,

$\nabla_{\xi_{i}} \varphi=0, \quad \nabla_{\xi_{i}} \xi_{j}=0$,

where $\nabla$ is the Levi Civita connection of $g$, $X \in \Gamma(T M)$ and $i, j \in\{1, \ldots s\}$ [8]. In 1995, Blair et al. [9] studied contact metric manifolds such that the characteristic vector field belongs to the $(\kappa, \mu)$-nullity distribution. This concept was generalized by Cappelletti-Montano and Di Terlizzi in [10].

\section{Preliminaries}

In this section, we give some fundamental informations which we use in the next part from [10].

Definition 2.1. Let $M$ be an almost $S$-manifold and let $\kappa, \mu$ be real constants. We say that $M$ verifies the $(\kappa, \mu)$ -nullity condition if and only if for each $i \in\{1, \ldots, s\}$ and $X, Y \in \Gamma(T M)$, the following identity holds

$R(X, Y) \xi_{i}=$

$+\kappa\left\{\sum_{k=1}^{s} \eta^{k}(X) \varphi^{2} Y-\eta^{k}(Y) \varphi^{2} X\right\}$

$+\mu\left\{\sum_{k=1}^{s} \eta^{k}(Y) h_{i} X-\eta^{k}(X) h_{i} Y\right\}$.

Lemma 2.1. Let $M$ be an almost $S$-manifold verifying the $(\kappa, \mu)$-nullity condition. Then we have

i) $h_{i} \circ h_{j}=h_{j} \circ h_{i}$ for each $i, j \in\{1, \ldots s\}$,

ii) $\kappa \leq 1$,

iii) if $\kappa<1$ then for each $i \in\{1, \ldots s\}, h_{i}$ has eigenvalues $0, \pm \sqrt{1-\kappa}$.

iv) $h_{i}^{2}=(\kappa-1) \varphi^{2}$.

Propositions 2.1. Let $M$ be an almost $S$-manifold verifying the $(\kappa, \mu)$-nullity condition. Then

$h_{1}=\ldots=h_{s}$.

Remark 2.1. Throughout all this paper whenever (2.1) holds we put $h:=h_{1}=\ldots=h_{s}$. Then (2.1) becomes 


$$
\begin{aligned}
& R(X, Y) \xi_{i}= \\
& +\kappa\left\{\sum_{k=1}^{s} \eta^{k}(X) \varphi^{2} Y-\eta^{k}(Y) \varphi^{2} X\right\} \\
& +\mu\left\{\sum_{k=1}^{s} \eta^{k}(Y) h X-\eta^{k}(X) h Y\right\} .
\end{aligned}
$$

Proposition 2.2. Let $M$ be an almost $S$-manifold verifying the $(\kappa, \mu)$-nullity condition. Then $M$ is an $S$-manifold if and only if $\kappa=1$.

\section{Weakly Symmetric Almost $S$-manifolds}

Firstly, we recall the definition of weakly symmetric manifolds.

Definition 3.1. A non-flat $n$-dimensional differential manifold $(M, g), n>3$, is called weakly symmetric if there exists a vector field $P$ and 1 -forms $\alpha, \beta, \gamma, \delta$ on $M$ such that

$\left(\nabla_{X} R\right)(Y, Z) W=\alpha(X) R(Y, Z) W$

$+\beta(Y) R(X, Z) W+\gamma(Z) R(Y, X) W$

$+\delta(W) R(Y, Z) X+g(R(Y, Z) W, X) P$,

holds for all vector fields $X, Y, Z, W \in \Gamma(T M)$. A weakly symmetric manifold $M$ is pseudo-symmetric if $\beta=\gamma=\delta=\frac{1}{2} \alpha \quad$ and $\quad P=A, \quad$ where $g(X, A)=\alpha(A)$ and $M$ is locally symmetric if $\alpha=\beta=\gamma=\delta=0$ and $P=0$. A weakly symmetric manifold is said to be proper if at least one of the 1 -forms $\alpha, \beta, \gamma, \delta$ are not zero or $P \neq 0[11,12]$.

From (3.1), an easy calculation shows that if $M$ is weakly symmetric then we have $\left(\nabla_{X} S\right)(Z, W)=\alpha(X) S(Z, W)$

$+\beta(R(X, Z) W)+\gamma(Z) S(X, W)$

$+\delta(W) S(X, Z)+P(R(X, W) Z)$,

where $P$ is defined by $\rho(X)=g(X, P)$ for any vector field $X \in \Gamma(T M)$ [11].

Now, we consider this definition for almost $S$-manifold verifying the $(\kappa, \mu)$-nullity condition.

Theorem 3.1. There exists no weakly symmetric $(2 n+s)$ -dimensional almost $S$-manifold $M$ verifying the $(\kappa, \mu)$ -nullity condition, if

$\alpha(X)+\gamma(X)+\delta(X) \neq \frac{X(\kappa)}{\kappa}$,

$X \in \Gamma(T M)$, where $\kappa$ is a smooth function and $\operatorname{dim}(M)>3$.

Proof. Assume that $M$ is a weakly symmetric almost $S$ manifold verifying the $(\kappa, \mu)$-nullity condition. Putting $W=\xi_{i}$ in (3.2), then we get

$\left(\nabla_{X} S\right)\left(Z, \xi_{i}\right)=\alpha(X) S\left(Z, \xi_{i}\right)$

$+\beta\left(R(X, Z) \xi_{i}\right)+\gamma(Z) S\left(X, \xi_{i}\right)$

$+\delta\left(\xi_{i}\right) S(X, Z)+P\left(R\left(X, \xi_{i}\right) Z\right)$.

By using $Q \xi_{i}=2 n \kappa \sum_{k=1}^{s} \xi_{k}$ then we conclude

$$
\begin{aligned}
& \left(\nabla_{X} S\right)\left(Z, \xi_{i}\right)=2 n \kappa \alpha(X) \sum_{k=1}^{s} \eta^{k}(Z) \\
& +\beta\left(R(X, Z) \xi_{i}\right)+2 n \kappa \gamma(Z) \sum_{k=1}^{s} \eta^{k}(X) \\
& +\delta\left(\xi_{i}\right) S(X, Z)+P\left(R\left(X, \xi_{i}\right) Z\right) .
\end{aligned}
$$

By the covariant differentiation of the Ricci tensor $S$, the left side of the above equation can be written as

$\left(\nabla_{X} S\right)\left(Z, \xi_{i}\right)=\nabla_{X} S\left(Z, \xi_{i}\right)$

$-S\left(\nabla_{X} Z, \xi_{i}\right)-S\left(Z, \nabla_{X} \xi_{i}\right)$.

Using (1.4) and $Q \xi_{i}=2 n \kappa \sum_{k=1}^{s} \xi_{k}$ and in view of the parallelity of the metric tensor $g$, then we derive

$$
\begin{aligned}
& \left(\nabla_{X} S\right)\left(Z, \xi_{i}\right)=2 n X(\kappa) \sum_{k=1}^{s} \eta^{k}(Z) \\
& -2 n \kappa g(Z, \varphi X)-2 n \kappa g(Z, \varphi h X) \\
& +S(Z, \varphi X)+S(Z, \varphi h X) .
\end{aligned}
$$

Comparing the right hand sides of the (3.4) and (3.5), we see that 
Celal Bayar University Journal of Science

$$
\begin{aligned}
& 2 n X(\kappa) \sum_{k=1}^{s} \eta^{k}(Z)-2 n \kappa g(Z, \varphi X) \\
& -2 n \kappa g(Z, \varphi h X)+S(Z, \varphi X) \\
& +S(Z, \varphi h X)=2 n \kappa \alpha(X) \sum_{k=1}^{s} \eta^{k}(Z) \\
& +\beta\left(R(X, Z) \xi_{i}\right)+2 n \kappa \gamma(Z) \sum_{k=1}^{s} \eta^{k}(X) \\
& +\delta\left(\xi_{i}\right) S(X, Z)+P\left(R\left(X, \xi_{i}\right) Z\right) .
\end{aligned}
$$

Now, putting $X=Z=\xi_{i}$ in (3.6) and using (1.1),

(1.2) and $Q \xi_{i}=2 n \kappa \sum_{k=1}^{s} \xi_{k}$, then we get

$2 n \xi_{i}(\kappa)=2 n \kappa \alpha\left(\xi_{i}\right)+2 n \kappa \gamma\left(\xi_{i}\right)+2 n \kappa \delta\left(\xi_{i}\right)$.

Since $\operatorname{dim} M>3$ and $\kappa \neq 0$, then we have

$$
\alpha\left(\xi_{i}\right)+\gamma\left(\xi_{i}\right)+\delta\left(\xi_{i}\right)=\frac{\xi_{i}(\kappa)}{\kappa} .
$$

Now, we will show that

$$
\alpha(X)+\gamma(X)+\delta(X)=\frac{X(\kappa)}{\kappa}
$$

for any vector field $X$ on $M$.

Setting $Z=\xi_{i}$ in (3.2), similar to the previous calculations, it follows that

$$
\begin{aligned}
& 2 n X(\kappa) \sum_{k=1}^{s} \eta^{k}(W)-2 n \kappa g(W, \varphi X) \\
& -2 n \kappa g(W, \varphi h X)+S(W, \varphi X) \\
& +S(W, \varphi h X)=2 n \kappa \alpha(X) \sum_{k=1}^{s} \eta^{k}(W) \\
& +\beta\left(R\left(X, \xi_{i}\right) W\right)+\gamma\left(\xi_{i}\right) S(X, W) \\
& +2 n \kappa \delta(W) \sum_{k=1}^{s} \eta^{k}(X)+P\left(R(X, W) \xi_{i}\right) .
\end{aligned}
$$

Replacing $W$ by $\xi_{i}$ in (3.8) and using (1.1) and

$$
\begin{aligned}
& Q \xi_{i}=2 n \kappa \sum_{k=1}^{s} \xi_{k} \text {, then we obtain } \\
& 2 n X(\kappa)=2 n \kappa \alpha(X) \\
& +\beta\left(R\left(X, \xi_{i}\right) \xi_{i}\right)+2 n \kappa \gamma\left(\xi_{i}\right) \sum_{k=1}^{s} \eta^{k}(X) \\
& +2 n \kappa \delta\left(\xi_{i}\right) \sum_{k=1}^{s} \eta^{k}(X)+P\left(R\left(X, \xi_{i}\right) \xi_{i}\right) .
\end{aligned}
$$

\section{Weakly Ricci Symmetric Almost $S$-manifolds}

Firstly, we recall the definition of weakly Ricci symmetric manifold and then we consider this definition for almost $S$ 
-manifolds.

Definition 4.1. An $n$-dimensional differential manifold $(M, g), n>3$, is called weakly Ricci symmetric if there exist 1 -forms $\varepsilon, \sigma, \rho$ on $M$ such that

$\left(\nabla_{X} S\right)(Y, Z)=\varepsilon(X) S(Y, Z)$

$+\sigma(Y) S(X, Z)+\rho(Z) S(X, Y)$

holds for all vector fields $X, Y, Z \in \Gamma(T M)[12,13]$. If $\varepsilon=\sigma=\rho$, then $M$ is called pseudo Ricci symmetric $[14,15]$.

Theorem 4.1. There exists no weakly Ricci symmetric almost $S$-manifold $M$ verifying the $(\kappa, \mu)$-nullity condition,

if $\varepsilon(X)+\sigma(X)+\rho(X) \neq \frac{X(\kappa)}{\kappa}$,

$X \in \Gamma(T M)$, where $\kappa$ is a smooth function and $\operatorname{dim}(M)>3$.

Proof. Let $M$ be a weakly Ricci symmetric almost $S$ manifold verifying the $(\kappa, \mu)$-nullity condition. Putting $Z=\xi_{i}$ in (4.1) and using $Q \xi_{i}=2 n \kappa \sum_{k=1}^{s} \xi_{k}$, then we obtain

$$
\begin{aligned}
& \left(\nabla_{X} S\right)\left(Y, \xi_{i}\right)=2 n \kappa \varepsilon(X) \sum_{k=1}^{s} \eta^{k}(Y) \\
& +2 n \kappa \sigma(Y) \sum_{k=1}^{s} \eta^{k}(X)+\rho\left(\xi_{i}\right) S(X, Y) .
\end{aligned}
$$

Replacing $Z$ by $Y$ in (3.5) and comparing the right hand sides of the equations (4.2) and (3.5), then we get

$$
\begin{aligned}
& 2 n X(\kappa) \sum_{k=1}^{s} \eta^{k}(Y)-2 n \kappa g(Y, \varphi X) \\
& -2 n \kappa g(Y, \varphi h X)+S(Y, \varphi X) \\
& +S(Y, \varphi h X)=2 n \kappa \varepsilon(X) \sum_{k=1}^{s} \eta^{k}(Y) \\
& +2 n \kappa \sigma(Y) \sum_{k=1}^{s} \eta^{k}(X)+\rho\left(\xi_{i}\right) S(X, Y) .
\end{aligned}
$$

Taking $X=Z=\xi_{i}$ in (4.3) and using (1.1), (1.2) and $Q \xi_{i}=2 n \kappa \sum_{k=1}^{s} \xi_{k}$ then we have $2 n \kappa\left\{\varepsilon\left(\xi_{i}\right)+\sigma\left(\xi_{i}\right)+\rho\left(\left(\xi_{i}\right)\right)\right\}=2 n \xi_{i}(\kappa)$.
Since $\operatorname{dim} M>3$ and $\kappa \neq 0$, we derive

$\varepsilon\left(\xi_{i}\right)+\sigma\left(\xi_{i}\right)+\rho\left(\left(\xi_{i}\right)\right)=\frac{\xi_{i}(\kappa)}{\kappa}$.

Putting $X=\xi_{i}$ in (4.3), then we get

$2 n \xi_{i}(\kappa) \sum_{k=1}^{s} \eta^{k}(Y)=2 n \kappa \varepsilon\left(\xi_{i}\right) \sum_{k=1}^{s} \eta^{k}(Y)$

$+2 n \kappa \sigma(Y)+2 n \kappa \rho\left(\xi_{i}\right) \sum_{k=1}^{s} \eta^{k}(Y)$.

Thus, from (4.4), this yields

$2 n \xi_{i}(\kappa) \sum_{k=1}^{s} \eta^{k}(Y)=2 n \kappa\left\{\varepsilon\left(\xi_{i}\right) \sum_{k=1}^{s} \eta^{k}(Y)\right.$

$\left.+\sigma(Y)+\rho\left(\xi_{i}\right) \sum_{k=1}^{s} \eta^{k}(Y)\right\}$

which gives us

$\sigma(Y)=\sigma\left(\xi_{i}\right) \sum_{k=1}^{s} \eta^{k}(Y)$.

Similarly, setting $Y=\xi_{i}$ in (4.3), we also obtain

$2 n X(\kappa)=2 n \kappa \varepsilon(X)$

$+2 n \kappa \sigma\left(\xi_{i}\right) \sum_{k=1}^{s} \eta^{k}(X)$

$+2 n \kappa \rho\left(\xi_{i}\right) \sum_{k=1}^{s} \eta^{k}(X)$.

Applying (4.4) into the last equation, then we deduce

$\varepsilon(X)=\frac{X(\kappa)}{\kappa}-\sum_{k=1}^{s} \eta^{k}(X)\left\{\frac{\xi_{i}(\kappa)}{\kappa}-\varepsilon\left(\xi_{i}\right)\right\}$.

Takiing $X=Y=\xi_{i}$ in (4.1), it follows that

$2 n \xi_{i}(\kappa) \sum_{k=1}^{s} \eta^{k}(Z)=$

$2 n \kappa\left\{\varepsilon\left(\xi_{i}\right)+\sigma\left(\xi_{i}\right)+\rho(Z)\right\}$.

By making use of (4.4), the equation (4.7) reduces to

$\rho(Z)=\sum_{k=1}^{s} \eta^{k}(Z) \rho\left(\xi_{i}\right)$.

Replacing $Y$ by $X, Z$ by $X$ in (4.5) and in (4.8), respectively and taking summation with (4.6), then we have

$\varepsilon(X)+\sigma(X)+\rho(X)=\frac{X(\kappa)}{\kappa}$,

for all vector field $X$. Hence, the proof is completed. 
Corollary 4.1. There exists no weakly Ricci symmetric almost $S$-manifolds $M$ verifying $(\kappa, \mu)$-nullity distribution, if $\varepsilon+\sigma+\rho \neq 0$, where $\kappa$ is a constant and $\operatorname{dim} M>3$.

\section{$5 \varphi$-symmetric Almost $S$-manifolds}

Definition 5.1. An almost $S$-manifold $M$, which verifies the $(\kappa, \mu)$-nullity condition, is said to be locally $\varphi$-symmetric if the

$$
\varphi^{2}\left(\nabla_{W} R(X, Y) Z\right)=0 \text {, }
$$

for all vector fields $X, Y, Z, W$ orthogonal to $\xi_{i}$, for each $i \in\{1, \ldots, s\}$. If the equation (5.1) is satisfied for arbitrary vector fields $X, Y, Z, W$, then $M$ is said to be $\varphi$-symmetric.

These notions were introduced for Sasakian manifolds by Takahashi in [11].

Theorem 5.1. A $\varphi$-symmetric almost $S$-manifold $M$ verifying the $(\kappa, \mu)$-nullity condition is an $\eta$-Einstein manifold of globally framed type.

Proof. By using (1.1) and (5.1), then we get

$$
-\left(\nabla_{W} R\right)(X, Y) Z
$$$$
+\sum_{k=1}^{s} \eta^{k}\left(\left(\nabla_{W} R\right)(X, Y) Z\right) \xi_{k}=0,
$$

from which it follows that

$$
\begin{aligned}
& -g\left(\left(\nabla_{W} R\right)(X, Y) Z, U\right) \\
& +\sum_{k=1}^{s} \eta^{k}\left(\left(\nabla_{W} R\right)(X, Y) Z\right) \eta^{k}(U)=0,
\end{aligned}
$$

for any vector field $U$ on $M$. Let

$$
\left\{e_{1}, \ldots, e_{2 n}, e_{2 n+1}=\xi_{1}, \ldots, e_{2 n+s}=\xi_{s}\right\}
$$

be an orthonormal basis of the tangent space at any point of the manifold. Then putting $X=U=e_{i}$ in (5.3) and taking summation over $1 \leq i \leq 2 n+s$, then we conclude

$$
\begin{aligned}
& -\left(\nabla_{W} S\right)(Y, Z) \\
& +\sum_{j=1}^{2 n+s} \eta^{j}\left(\left(\nabla_{W} R\right)\left(e_{i}, Y\right) Z\right) \eta^{j}\left(e_{i}\right)=0 .
\end{aligned}
$$

The second term of (5.4) by putting $Z=\xi_{j}$ takes the form $\eta^{j}\left(\left(\nabla_{W} R\right)\left(e_{i}, Y\right) \xi_{k}\right) \eta^{j}\left(e_{i}\right)$ $=g\left(\left(\nabla_{W} R\right)\left(e_{i}, Y\right) \xi_{j}, \xi_{j}\right) g\left(e_{i}, \xi_{j}\right)$,
$g\left(\left(\nabla_{W} R\right)\left(e_{i}, Y\right) \xi_{j}, \xi_{j}\right)=$

$+g\left(\nabla_{W} R\left(e_{i}, Y\right) \xi_{j}, \xi_{j}\right)$

$-g\left(R\left(\nabla_{W} e_{i}, Y\right) \xi_{j}, \xi_{j}\right)$

$-g\left(R\left(e_{i}, \nabla_{W} Y\right) \xi_{j}, \xi_{j}\right)$

$$
-g\left(R\left(e_{i}, Y\right) \nabla_{W} \xi_{j}, \xi_{j}\right)
$$

at $p \in M$. Since the basis is orthonormal, thus we have $\nabla_{W} e_{i}=0$ at $p \in M$. Using (1.1), (1.3) and (2.3), then we get

$g\left(R\left(e_{i}, \nabla_{W} Y\right) \xi_{j}, \xi_{j}\right)=0$.

Taking into account of (5.7) in (5.6), then we derive

$g\left(\left(\nabla_{W} R\right)\left(e_{i}, Y\right) \xi_{j}, \xi_{j}\right)=$

$+g\left(\nabla_{W} R\left(e_{i}, Y\right) \xi_{j}, \xi_{j}\right)$

$-g\left(R\left(e_{i}, Y\right) \nabla_{W} \xi_{j}, \xi_{j}\right)$,

Since $\quad g\left(R\left(e_{i}, Y\right) \xi_{j}, \xi_{j}\right)=-g\left(R\left(\xi_{j}, \xi_{j}\right) Y, e_{i}\right)=0$ hence we get

$g\left(\nabla_{W} R\left(e_{i}, Y\right) \xi_{j}, \xi_{j}\right)+g\left(R\left(e_{i}, Y\right) \xi_{j}, \nabla_{W} \xi_{j}\right)=0$.

Using the last equation in (5.8), then we have

$$
\begin{aligned}
& g\left(\left(\nabla_{W} R\right)\left(e_{i}, Y\right) \xi_{j}, \xi_{j}\right)=-g\left(R\left(e_{i}, Y\right) \xi_{j}, \nabla_{W} \xi_{j}\right) \\
& -g\left(R\left(e_{i}, Y\right) \nabla_{W} \xi_{j}, \xi_{j}\right) .
\end{aligned}
$$

From (1.4) and first Bianchi identity, we obtain

$g\left(\left(\nabla_{W} R\right)\left(e_{i}, Y\right) \xi_{j}, \xi_{j}\right)=g\left(R\left(e_{i}, Y\right) \xi_{j}, \varphi W\right)$

$+g\left(R\left(e_{i}, Y\right) \xi_{j}, \varphi h W\right)+g\left(R\left(e_{i}, Y\right) \varphi W, \xi_{j}\right)$

$+g\left(R\left(e_{i}, Y\right) \varphi h W, \xi_{j}\right)=0$,

i.e.,

$g\left(\left(\nabla_{W} R\right)\left(e_{i}, Y\right) \xi_{j}, \xi_{j}\right)=0$.

Using (5.9) in (5.4), then we deduce

$\left(\nabla_{W} S\right)\left(Y, \xi_{j}\right)=0$.

It is well-known that

$\left(\nabla_{W} S\right)\left(Y, \xi_{j}\right)=\nabla_{W} S\left(Y, \xi_{j}\right)$

$-S\left(\nabla_{W} Y, \xi_{j}\right)-S\left(Y, \nabla_{W} \xi_{j}\right)$.

From (3.5), it follows that

$\left(\nabla_{W} S\right)\left(Y, \xi_{i}\right)=2 n W(\kappa) \sum_{k=1}^{s} \eta^{k}(Y)$

$-2 n \kappa g(Y, \varphi W)-2 n \kappa g(Y, \varphi h W)$

$+S(Y, \varphi W)+S(Y, \varphi h W)$.

Replacing $Y$ by $\varphi Y$ in (5.11), then we have 


$$
\begin{aligned}
& S(Y, W)=\{s(2(n-1)+\mu)(\kappa-1) g(Y, W)\} \\
& +4 n \kappa \sum_{k=1}^{s} \eta^{k}(Y) \eta^{k}(W) .
\end{aligned}
$$

This completes the proof.

\section{$6 \varphi$-Ricci symmetric Almost $S$-manifolds}

Definition 6.1. An almost $S$-manifold $M$ verifying the $(\kappa, \mu)$-nullity condition is said to be $\varphi$-Ricci symmetric if the Ricci operator $Q$ satisfies

$$
\varphi^{2}\left(\nabla_{X} Q\right) Y=0 \text {, }
$$

any vector fields $X$ and $Y$ on $M$ and $S(X, Y)=g(Q X, Y)$. If $X$ and $Y$ are orthogonal to $\xi_{i}$ for each $i \in\{1, \ldots, s\}$, then $M$ is called locally $\varphi$ Ricci symmetric.

Now, we give the definition of Einstein manifold of globally framed type.

Definition 6.2. An almost $S$-manifold $M$ verifying the $(\kappa, \mu)$-nullity condition is said to be $\eta$-Einstein manifold of globally framed type if its Ricci tensor $S$ is of the form $S(X, Y)=A g(X, Y)+B \sum_{k=1}^{s} \eta^{k}(X) \eta^{k}(Y)$,

where $A$ and $B$ are smooth function and $X$ and $Y$ are vector fields on $M$.

Theorem 6.1. An almost $S$-manifold $M$ verifying the $(\kappa, \mu)$-nullity condition is an $\eta$-Einstein manifold of globally framed type, if

$$
\mu=2\left(1-\frac{1}{n}+\frac{\kappa}{s}\right) \text { or the }(1,1) \text {-tensor field } h
$$

vanishes identically.

Proof. In view of the assumption, using (1.1) in (6.1), then we have

$$
-\left(\nabla_{X} Q\right) Y+\sum_{k=1}^{s} \eta^{k}\left(\left(\nabla_{X} Q\right) Y\right) \xi_{k}=0 .
$$

Taking the inner product of (6.2) with $Z$, then we obtain

$$
-g\left(\left(\nabla_{X} Q\right) Y, Z\right)+\sum_{k=1}^{s} \eta^{k}\left(\left(\nabla_{X} Q\right) Y\right) \eta^{k}(Z)=0 \text {, }
$$

which on simplifying gives us

$$
\begin{aligned}
& -g\left(\nabla_{X} Q Y, Z\right)+S\left(\nabla_{X} Y, Z\right) \\
& +\sum_{k=1}^{s} \eta^{k}\left(\left(\nabla_{X} Q\right) Y\right) \eta^{k}(Z)=0 .
\end{aligned}
$$

Taking $Y=\xi_{i}$ in (6.3), then we derive

$$
\begin{aligned}
& -g\left(\nabla_{X} Q \xi_{i}, Z\right)+S\left(\nabla_{X} \xi_{i}, Z\right) \\
& +\sum_{k=1}^{s} \eta^{k}\left(\left(\nabla_{X} Q\right) \xi_{i}\right) \eta^{k}(Z)=0 .
\end{aligned}
$$

Using (1.4) in (6.4), then we get

$$
\begin{aligned}
& -2 n X(\kappa) \sum_{k=1}^{s} \eta^{k}(Z)+2 n \kappa g(Z, \varphi X) \\
& -2 n \kappa g(Z, \varphi h X)-S(Z, \varphi X) \\
& -S(Z, \varphi h X)+\sum_{k=1}^{s} \eta^{k}\left(\left(\nabla_{X} Q\right) \xi_{i}\right) \eta^{k}(Z)=0 .
\end{aligned}
$$

Replacing $Z$ by $\varphi Z$ in (6.5) and using

$S(\varphi X, \varphi Z)=-S(X, Z)+2 n \kappa \sum_{k=1}^{s} \eta^{k}(X) \eta^{k}(Z)$,

then we conclude

$$
\begin{aligned}
& S(X, Z)=-\{2 n \kappa-s(2(1-n)+\mu n)\} g(h X, Z) \\
& -\{2 s(2(n-1)+\mu)(1-\kappa)+2 n \kappa\} g(X, Z) \\
& +\{4 n \kappa+s(2(1-n)+\mu n)\} \sum_{k=1}^{s} \eta^{k}(X) \eta^{k}(Z) .
\end{aligned}
$$

Thus, we complete the proof.

\section{Conclusion}

Symmetric manifolds play an important role in differential geometry. Thus, classification of manifolds with respect to symmetry properties helps the researcher for their advanced studies. We prove some existence theorems for some weakly symmetric manifolds. We give a characterization of $\varphi$-symmetric spaces.

\section{Acknowledgements}

This work was supported by the Düzce University Scientific Research Projects [Project number 2016.05.04.431].

\section{Referanslar}

1. Yano, K., On a structure $f$ satisfying $f^{3}+f=0$, Technical Report No. 12, University of Washington, USA, 1961.

2. Goldberg, S.I., Yano, K., Globally framed $f$-manifolds, Illinois Journal of Mathematics, 1971, 15(3), 456-474.

3. Ishihara, S., Normal structure $f$ satisfying $f^{3}+f=0$, Kodai Mathematical Seminar Reports, 1966, 18(1), 36-47.

4. Blair, D.E., Geometry of manifolds with structural group $U(n) \times O(s)$, Journal of Differential Geometry, 1970, 4(2), 155-157. 
5. Goldberg, S.I., Yano, K., On normal globally framed $f$-manifolds, Tohoku Mathematical Journal, 1970, 22, 362-370.

6. Vanzura, J., Almost $r$-contact structures, Annali della Scuola Normale Superiore di Pisa Mathématiques, 1972, 26, 97-115.

7. Cabrerizo, J.L., Fernandez, L.M., Fernandez, M., The curvature tensor fields on $f$-manifolds with complemented frames, Annals of the Alexandru Ioan Cuza University - Mathematics, 1990, 36, 151-161.

8. Duggal, K.L., Ianus, S., Pastore, A.M., Maps interchanging $f$-structures and their harmonicity, Acta Applicandae Mathematicae, 2001, 67(1), 91-115.

9. Blair, D.E., Koufogiorgos, T., Papantoniou, B.J., Contact metric manifolds satisfying a nullity condition, Israel Journal of Mathematics, 1995, 91, 189-214.

16.
10. Cappelletti-Montano, B., Di Terlizzi, L., $D$-homothetic transformations for a generalization of contact metric manifolds, Bulletin of the Belgian Mathematical Society - Simon Stevin, 2007, 14, 277-289.

11. Takahashi, T., Sasakian $\varphi$-symmetric space, Tohoku Mathematical Journal, 1977, 29, 91-113.

12. Tamassy, L., Binh, T.Q., On weak symmetries of Einstein and Sasakian manifolds, Tensor N.S. 1993, 53, 140-148.

13. Tamassy, L., Binh, T.Q., On weakly symmetric and weakly projective symmetric Riemannian manifolds, Colloquium Mathematical Society Janos Bolyai, 1992, 56, 663-670.

14. Chaki, M.C., On pseudo Ricci-symmetric manifolds, Bulgarian Journal of Physics, 1988, 15, 526-531.

15. Dileo, G., Lotta, A., On the structure and symmetry properties of almost $S$-manifolds, Geometriae Dedicata, 2005, 110, 191-211. 